\title{
Urgences
}

\section{Images d'angoisse : Tintin, l'araignée et le champignon}

\section{Jacques Samson}

Numéro 32, mai 1991

Lectures de bandes dessinées

URI : https://id.erudit.org/iderudit/025645ar

DOI : https://doi.org/10.7202/025645ar

Aller au sommaire du numéro

Éditeur(s)

Urgences

ISSN

0226-9554 (imprimé)

1927-3924 (numérique)

Découvrir la revue

Citer cet article

Samson, J. (1991). Images d'angoisse : Tintin, l'araignée et le champignon.

Urgences, (32), 18-29. https://doi.org/10.7202/025645ar d'utilisation que vous pouvez consulter en ligne.

https://apropos.erudit.org/fr/usagers/politique-dutilisation/ 


\title{
Images d'angoisse: Tintin, l'araignée et le champignon Jacques Samson
}

\author{
à J.L. et P.C.
}

Album plein de malaises et de phénomènes étranges s'il en est, L'étoile mystérieuse d'Hergé témoigne d'une intrusion de Tintin, somme toute exceptionnelle, dans l'univers ténébreux et morbide de l'angoisse. La chose est entendue; plusieurs commentateurs ont en effet attiré l'attention sur la singularité de ce récit créé, il n'est pas inutile de le rappeler, sous l'occupation allemande de la Belgique, pendant la Seconde Guerre mondiale. Serge Tisseron, par exemple, en a proposé une lecture évoquant les diverses phases du processus de la création artistique ${ }^{1}$; Jean-Marie Apostolidès ${ }^{2}$ et Frédéric Soumois ${ }^{3}$ ont noté, chacun selon sa perspective propre, les accents apocalyptiques d'une aventure au sortir de laquelle la figure tintinienne aussi bien que l'esthétique d'Hergé ont connu de profondes transformations; enfin Benoît Peeters ${ }^{4}$ a mis l'accent, pour sa part, sur la grande originalité d'un récit qui énonce en son thème certaines modalités de son tracé structurel.

De mon côté, et sans rien retrancher aux commentaires auxquels il est ci-devant fait référence, j'aimerais mettre en relief un aspect du travail fantasmatique à l'œuvre dans cet album: je veux parler de la façon dont il s'acquitte de l'évocation de l'angoisse. Il me semble, au vu de l'incoercible émotion engendrée par certaines séquences d'images de L'étoile mystérieuse, que non seulement Hergé a pu accomplir, en créant cet album, une manière d'exorcisme en lien plus ou moins direct

\footnotetext{
1 "L'étoile mystérieuse: le récif de la création", dans Hergé, coll. "Les auteurs par la bande ", Paris, Le club des stars-Seghers, 1987, p. 27 à 49.

2 "La fin d'un monde", dans Les métamorphoses de Tintin, Paris, Seghers, 1984, p. 140 à 148.

3 "L'étoile mysténieuse, apocalypse aux champignons ", dans Dossier Tintin, Bruxelles, Jacques Antoine éditeur, 1987, p. 167 à 173.

4 "Microcosme et macrocosme", dans Les bijoux ravis, Bruxelles, Magic Strip, 1984, p. 12-13.
} 
avec les inquiétudes qu'il pouvait ressentir à l'époque, mais que le moyen artistique avec lequel il a choisi de s'exprimer la bande dessinée - s'est par là également enrichi d'un potentiel d'expressivité pour une bonne part inédit. Il est vrai que, pour peu qu'il ait affaire à des représentations ou à des sentiments aussi «investis" et intenses que les affects anxiogènes, l'auteur de bandes dessinées - comme n'importe quel autre artiste d'ailleurs - est souvent amené à transcender ses propres limites expressives en "créant pour un thème précis une forme spécifique", selon les mots de Soumois ${ }^{5}$. À cause précisément des particularités de sa thématique, L'étoile mysterieuse regorge de semblables dépassements formels à travers lesquels il est possible, à mon avis, de voir une explicitation sui generis de certains procédés formels de la bande dessinée.

Dans L'étoile mystérieuse, le monde paraît d'emblée assujetti à une logique hallucinatoire qui confronte Tintin à de terrifiantes anomalies et l'amène à douter de lui-même, plus précisément à douter de ses propres capacités perceptives. Tel un rêveur diurne, il évolue dans un univers chaotique qui n'a plus rien de familier à ses yeux. Rêve ou réalité? La distinction paraît plus délicate que jamais, comme dans ce rêve de la planche 9 qui revêt curieusement toutes les apparences de la réalité. Où qu'il se pose, le regard de Tintin n'en finit pas de croiser des phénomènes inattendus qui le somment d'exprimer, tant bien que mal, sa surprise ou son malaise: "C'est extraordinaire! [...] Je n'en reviens pas! [...] Ça m'intrigue. [...] Je n'ai pourtant pas la berlue ! [...] Tout cela est bien étrange ! En amorce du récit, un clivage a tôt fait de s'établir entre les sensations qu'éprouve Tintin, ses attentes en regard de la réalité et le monde détraqué qui l'environne brusquement. Rarement a-t-on vu Tintin aussi dérouté, aussi frappé de stupeur, aussi manifestement saisi de débordements émotionnels. Quand ce n'est pas la peur ou l'angoisse qui s'empare de lui, il est transporté d'une joie aussi soudaine qu'excessive ( $\mathrm{pl}$. 10 et 13). Inexplicables mouvements d'humeur qui font d'ailleurs croire à Milou que son maître n'est pas loin d'être dément ( $\mathrm{Il}$ est complètement marteau "). 
Dès la quatrième planche, un événement bouleversant d'une intensité qu'on pourrait même croire traumatisante ${ }^{6}$ survient, qui ne manquera pas de laisser son empreinte sur le reste du récit: Tintin aperçoit très distinctement à travers la lunette de l'Observatoire - aussi distinctement que nousmêmes d'ailleurs - une monstrueuse araignée noire dont la taille gigantesque et les " pattes velues " lui font pousser des cris d'horreur et d'effroi... « Mon dieu!... C'est horrible! [...] Rien que d'y songer me donne le frisson!» Témoin sceptique de la scène, le professeur Hippolyte Calys se défie aussitôt de la perception de Tintin et redoute que le jeune homme ait l'esprit dérangé: "Une araignée? Est-ce que vous n'en avez pas une dans le plafond? " C'en est trop pour Tintin qui, s'empressant d'aller vérifier la chose, comprend immédiatement sa méprise et se rassérène; une trompeuse illusion d'optique a démesurément grossi la "petite araignée de rien du tout" (aux dires de Milou) qui s'était posée, selon toute vraisemblance, au centre de l'objectif du télescope. Pourtant, sitôt le personnage rassuré, les visions d'horreur recommencent, ou plutôt se poursuivent de plus belle... Une meute de rats comme "frappés de panique" quitte les égouts. Sous l'action excessive de la chaleur, les pneus des voitures éclatent et l'asphalte fond. Un vieillard cinglé (Philippulus), aperçu peu avant dans l'Observatoire, prophétise à grands coups de gong la fin du monde. Rien ne va plus. Les certitudes de Tintin s'effondrent aussi aisément et fatalement que les murs et le plafond de son appartement secoué par un violent séisme... Décidément, cette histoire commence bien mal. Jamais, pense-t-on, une aventure de Tintin n'aura été placée sous une si mauvaise étoile.

Abandonnons là le fil du récit et attardons-nous davantage sur la nature des phénomènes qui troublent tant notre héros, d'habitude si sûr de lui, si conscient de ses valeurs et de ses capacités, si confiant en son jugement. D'une certaine manière, il n'est pas exagéré de prétendre que l'inquiétant dérèglement du monde auquel est exposé Tintin en début du récit, l'affecte jusque dans son jugement et ses perceptions. Il

6 Le retour obsédant et terrifiant de l'araignée - dans le texte (pl. 50, 53 et 54) et dans l'image (pl. 54 et 55) - donne l'exacte mesure de l'intensité de l'émotion ressentie alors par Tintin. 
intériorise des phénomènes extérieurs à lui, jusqu'à les faire siens, exactement comme s'ils étaient le fruit de sa propre imagination. Ce monde en proie à la dévastation, il en fait d'emblée une affaire personnelle. À l'instar de l'univers environnant qui se démantèle de toutes parts et dont on annonce la fin cataclysmique, Tintin pressent qu'il est lui-même voué à la catastrophe. C'est peut-être d'ailleurs la crainte d'un immense désastre intérieur qui le préoccupe au plus haut point? En effet, la position centrale qu'il occupe dans ce monde au bord de l'abîme en fait à la fois le témoin privilégié et la victime essentielle. Principal actant du drame - héros qui serait tout à coup incapable de maintenir en état l'univers qui lui sert de territoire épique -, il est accablé par un savoir que ne partagent pas les gens ordinaires («Pauvres gens! S'ils savaient!») et montre un affolement auquel paraissent insensibles les savants, tant ils sont préoccupés par leurs calculs. De fait, Tintin est si profondément troublé par ce qu'il sait et observe autour de lui qu'il en est perturbé sensoriellement, en particulier sur le plan de la vision. S'il n'est certes pas insensible au dérèglement climatique, cela paraît secondaire en comparaison de tout ce dont il est le témoin oculaire direct. C'est d'abord et essentiellement par l'entremise du regard qu'il prend en effet connaissance de ce qui ne tourne pas rond dans le monde effrayant qu'il habite.

Une chose frappe tout particulièrement ici: la terreur de loin la plus vive éprouvée jusque là par Tintin -- la vision cauchemardesque de l'araignée géante - est le fruit d'un leurre, d'une tromperie de ses sens (figure 1). Ainsi, à l'Observatoire, s'il voit bien l'araignée - nul doute là-dessus! il se montre pourtant totalement inapte à en évaluer correctement la taille; sur le moment, il est incapable de saisir l'effet d'illusion par grossissement que lui renvoie la lunette astronomique. Ce qui provoque en lui une angoisse sans nom résulte donc bel et bien d'une erreur de sa part dans l'appréciation des proportions. À la vérité, ce sont les capacités perceptives de Tintin qui sont en jeu ici. Une «clarté aveuglante " ${ }^{7}$ l'empêche d'aller au bout de son regard, c'est-à-dire

7 Remarquons que la « clarté aveuglante " dont parle Tintin (pl. 8) figure très nettement en arrière-plan de l'araignée géante, dans la vue du téléscope. Un lien visuel est donc établi entre l'araignée et l'étoile mystérieuse... 

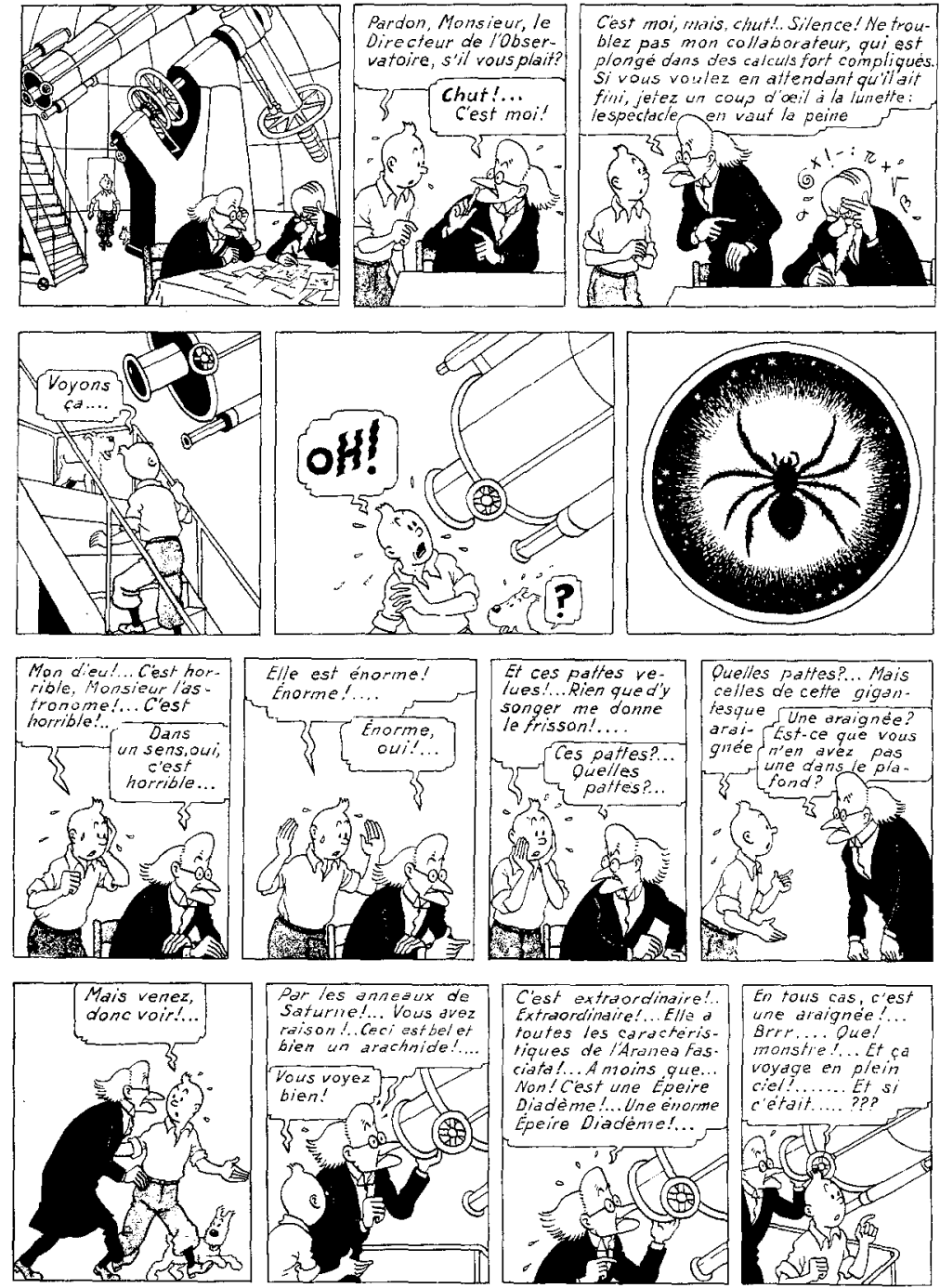

Figure 1 
jusqu'à l'intelligence complète et réelle de la chose perçue. Avec sa perception des choses déjà considérablement ébranlée par l'observation d'une étoile aux proportions démesurées et par le malaise d'une chaleur suffocante, Tintin omet un indice visuel capital en posant l'œil dans le télescope, un indice nettement suffisant - en principe - pour évaluer correctement la dimension de la chose vue et sa distance relative par rapport à lui: la taille familière de l'arachnide. Il devrait savoir et prendre en compte le fait que la très grande taille d'un petit objet familier suggère son rapprochement par rapport à un point d'observation donné, et vice versa. Mais ce n'est pas le cas. Le point de vue fait problème pour Tintin.

La source de cet imbroglio perceptif est double: sur un plan "objectif» d'abord, le télescope, l'appareil grossissant en tant que tel, agent proprement dit de la distorsion optique, et ensuite, sur un plan "subjectif", l'œil de Tintin qui ne sait pas ou ne peut pas interpréter de la bonne manière le gain perceptif enregistré par la rétine. La vue télescopique combinée à l'organe perceptif de Tintin génère, au sens fort du terme, une perception appréhensive qui revêt les allures d'une représentation fantasmatique. Il faut bien comprendre que le télescope ne déforme pas, à strictement parler, la représentation de l'araignée, il la restitue telle quelle, mais dans des proportions différentes. Alors que, pour l'œil de Tintin, l'image de la lunette renvoie une vision deformee, c'est-à-dire formée à partir d'un autre point de vue, d'une autre perspective, d'une autre scène qui refuse absolument la prise en compte, dans le champ visuel et mental, de l'effet transformationnel du télescope. On notera également qu'il n'y a pas chez Tintin défaut de représentation par rapport à cette image; si elle lui apparaît impensable, elle n'est pourtant pas à ses yeux totalement irreprésentable; il la voit bien mais elle lui fait l'effet d'une mauvaise image. Parce qu'elle est surinvestie et dotée d'un formidable quantum d'affect, l'image de l'araignée révèle un conflit chez Tintin entre le désir de voir - et même de voir mieux et davantage grâce à la lunette ${ }^{8}$ et la défense de voir - c'est-à-dire le refus de comprendre la

8 II faut noter à cet égard l'empressement de Tintin à faire mention de l'Obsenvatoire (dès la septième vignette de l'album) et à s'y rendre, comme si la levée de l'énigme impliquait d'ores et déjà l'amplification optique. 
vision pour ce qu'elle est: un fantasme archaïque. À la vérité, la vue angoissée de l'Observatoire préfigure déjà la rencontre avec le monstre de la planche 55 !

La scène de l'Observatoire est digne d'intérêt parce qu'elle constitue une exceptionnelle mise en image du principe de déformation à l'œuvre dans le processus de l'émotion anxiogène. Au moment où Tintin jette son regard dans la lunette, il mêle une part intime de sa vie imaginaire avec la réalité objective. En posant son œil dans le viseur du télescope - instrument amplificateur par excellence de la perception humaine - il quitte le domaine des observations normales et se met en situation de saisissement: craindre ce qu'il va voir ou voir ce qu'il craint le plus. Le télescope donne en effet accès à une représentation purement imaginaire parce que grossie démesurément, décuplée, sans commune mesure avec l'appréhension ordinaire des choses; par un jeu de franchissement optique analogue au miroir, il amène Tintin à projeter son regard au-dedans de lui-même, à quitter le monde des observations rationnelles et à se confronter à ses propres images d'angoisse.

En clair, la scène de l'Observatoire équivaut à une fantaisie régressive; elle ramène Tintin à un stade de perception prérationnel ou prédiscursif. Au lieu d'accéder à un ordre de représentation symbolique - où l'araignée serait associée à l'imaginaire sexuel primitif ${ }^{9}$, il ne sait exprimer que la démesure de sa perception obnubilée («Elle est énorme! Énorme!"). En schématisant, on peut énoncer en une formule simple la nature de ce fantasme régressif:

\begin{tabular}{|l||l|l|}
\hline Vision fantasmatique $=$ & Énorme araignée & Petit Tintin \\
\hline \hline Vision rationnelle* $=$ & Petite araignée & Énorme Tintin \\
\hline
\end{tabular}

* Représentation qui tient compte du jeu de l'amplification optique.

Il est évident, encore une fois, que l'émotion anxiogène provient de ce que Tintin échoue à ramener les choses à leurs justes proportions; l'énormité de l'araignée ne peut le renvoyer qu'à sa petitesse. Tintin craint désespérément d'être la

9 A dire vrai, il y a dans la parole de Tintin une allusion à peine voilée à cette sorte de représentation sexuelle primitive: " Et ces pattes velues!... ” 
proie de l'arachnide. La vue de l'araignée provoque en lui une effroyable angoisse de morcellement, de dévoration ou d'annihilation.

Du point de vue de la lecture, la réalité donnée à voir se moule d'après les perceptions erronées de Tintin. Par conséquent, le lecteur est lui aussi la proie d'une illusion, l'illusion de l'image donnée à voir comme la vue de Tintin. En un sens, l'image de l'Épeire Diadème devrait pouvoir être interprétée isolément et disons "littéralement", c'est-à-dire suivant les données comparatives du réel, soit comme une toute petite araignée démesurément agrandie par la lunette télescopique. Mais cette lecture supposerait une mise à distance de la fiction, fort peu vraisemblable dans les circonstances ${ }^{10}$, et ce sans compter que, pour grand nombre de gens, l'araignée appartient à la catégorie des animaux d'angoisse fortement surinvestis en termes fantasmatiques.

En ce qui concerne l'organisation du matériel signifiant, la scène de l'Observatoire constitue une remarquable mise à contribution des capacités métaphoriques de la bande dessinée. Pour fonctionner efficacement, les deux vignettes centrales de cette scène (images 5 et 6 de la pl. 4) doivent momentanément échapper à la règle de la consécution et entrer dans une relation de pure contiguité; elles glissent de la logique des continuités à celle des points de vue simultanés, logique qui confere à la seconde vignette (la vue de l'araignée à travers le télescope) le statut de contrechamp par rapport à la première (le regard de Tintin). Ainsi, par le jeu d'une substitution dans l'ordre du paradigme, la vision lectorielle devient l'objet d'un transfert interprétatif: aux yeux du lecteur, la vue de l'araignée est donnée en lieu et place du regard de Tintin. Il y a donc impossibilité de se soustraire au fantasme de Tintin. Ici la bande dessinée est mise en scène et mise en acte du fantasme: elle mêle à la fois la vision fantasmatique (la vue de l'araignée géante) et le regard fantasmé (le point de vue de Tintin). Le procès métaphorique à l'œuvre dans ces images lie très étroitement le voir et le savoir, de façon à placer Tintin et le lecteur exactement sur le même pied. Le génie de Hergé consiste à avoir imaginé une figuration de

10 Comme on va le voir ci-après, le dispositif narratif outrepasse largement le cadre même de la diégèse. 
l'émotion anxiogène qui tienne parfaitement compte de sa mécanique déformante, en usant de l'amplification optique comme d'une métaphore de ce mécanisme lui-même.

Du reste, on observera également que le recours à l'appareil d'optique représenté par la lunette astronomique, inscrit d'entrée de jeu le récit d'Hergé dans un univers fantasmagorique générateur d'inquiétante étrangeté, ainsi que l'a bien démontré Max Milner ${ }^{11}$.

L'appareil d'optique, qui permet au héros de déplacer son regard vers le lieu de son désir, a en effet ceci de particulier que ce qu'il donne à voir est affecté d'un coefficient d'incertitude permettant au narrateur de mettre en évidence aussi bien le statut problématique du réel que le caractère illusoire de l'imaginaire et de faire jouer l'un par rapport à l'autre deux espaces incompatibles, à l'intersection desquels le sens vacille et l'interprétation prolifère ${ }^{12}$.

Et c'est bien l'une des particularités de L'étoile mystérieuse que de tramer en son récit une problématique du voir parfaitement compatible avec la mise en scène des appréhensions régressives de Tintin. Aucun doute n'est permis, c'est au non-visible - comme on parle de non-dit - que l'on a affaire dans cet extraordinaire récit...

Une autre scène illustre à merveille - et d'une façon plus convaincante encore peut-être que celle de l'Observatoire d'autres propriétés figurales de la bande dessinée. Il s'agit du dernier strip de la planche $51^{13}$ (figure 2), qui montre Tintin et Milou regardant stupéfaits la croissance progressive et colossale d'un champignon (il faut dire, d'abord perçu comme un œuf). La scène se déroule sur l'aérolithe - la mauvaise étoile - et fait massivement retour à la scène de l'Observatoire

11 Max Miner, La fantasmagorie, coll. “Ecriture ”, Paris, PUF, 1982.

12 Ibid., p. 61.

13 II n'est pas interdit de penser qu'à l'origine le strip en question devait inclure la vignette précédente (à moins qu'il ne s'agisse de la suivante?) puisque, cormme on sait, L'étoile mystérieuse a d'abord paru sous forme de strips quotidiens, dans le quotidien belge Le soir, du 20 octobre 1941 au 21 mai 1942. Au moment d'en faire un album, Hergé a dû remanier considérablement son récit ainsi que l'a expliqué Soumois (op. cit., p. 167). Pour ce qui va suivre, cela ne fait toutefois pas de différence puisque les lecteurs de l'album percoivent assurément ce strip comme une totalité et c'est ce qui importe. 

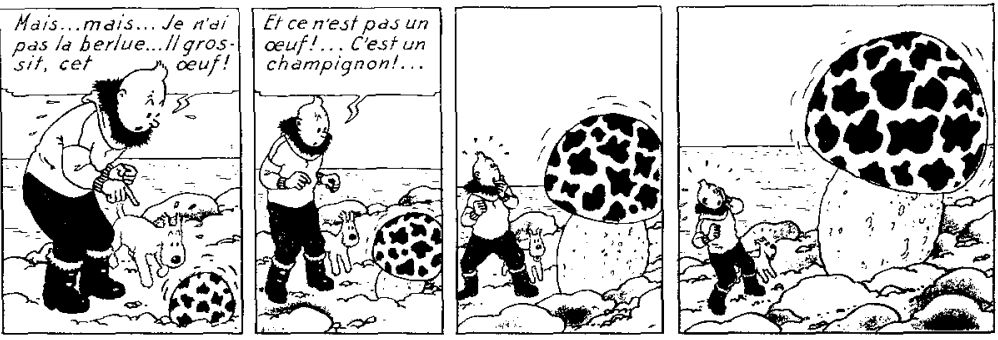

Figure 2

(réactivée auparavant dans le rêve de la planche 9). Les propriétés d'amplification de la lunette astronomique se trouvent ici transférées, par déplacement et condensation, à tous les objets environnants, ce qui projette Tintin et Milou au cœur du cauchemar insoutenable anticipé en début de récit. La logique de tous les jours fait alors défaut et le fantasme a acquis une telle amplitude, un tel paroxysme qu'il déforme absolument tout. Les métamorphoses diverses qu'il fait subir aux objets familiers évoquent toutes le cycle de la vie, la fécondité, à travers une symbolisation sexuelle on ne peut plus évidente.

En l'examinant de plus près, la scène du champignon a ceci de particulier - par rapport à celle de l'Observatoire - qu'elle distingue la vision de Tintin de celle du lecteur, et, par voie de conséquence, leurs savoirs respectifs. Si l'un et l'autre sont témoins de l'accroissement fantastique du champignon, le lecteur est toutefois amené, grâce à la singulière mise en images d'Hergé, à voir autre chose en surplus. À la façon dont sont représentées les quatre vignettes de la scène, comment pourrait-il en effet ne pas voir que la croissance du champignon est montrée dans un rapport directement proportionnel à la décroissance de Tintin ou bien encore qu'à l'agrandissement du champignon correspond, image pour image, le rapetissement de Tintin? Dans cette scène, ne dirait-on pas que la bande dessinée jouit soudain de propriétés analogues à celles de la lunette télescopique?

Alors que Tintin est confiné à la stricte continuité des images, le lecteur tire avantage d'une vision ambivalente de la scène, structurée par un double point de vue de lecture: la lecture linéaire d'abord, mettant à profit la consécution des vignettes, puis la lecture tabulaire, prenant acquis de leur contiguïté spatiale. Dans le premier cas, la lecture s'avère 
indissociable du continuum spatio-temporel, interne à la diégèse, tandis que la seconde libère un espace extra-diégétique, issu du procès narratif, inducteur de ce que j'ai ailleurs nommé une «lexie globalisante ou méta-point de vue de lecture" à travers quoi "le dispositif lui-même énonce, selon sa propre logique, une vérité d'images qui n'a de sens que dans la pure fiction de la lecture" 14 .

En termes picturaux, la parfaite cohérence du décryptage tabulaire tient pour une part appréciable à la faible présence de points de repère visuels assurant au lecteur l'interprétation univoque de la scène, c'est-à-dire selon le seul point de vue de Tintin. Dans le strip qui nous intéresse, l'ensemble du décor rocaille brunâtre en avant-plan, horizon bleu et mer verte en arrière-plan - fournit des indices visuels peu nombreux et différenciés qui peuvent aisément paraître fixes ou invariables, ce qui atténue ainsi fortement l'effet de progression scalaire inscrit dans la suite des images. On sait en effet que la densité des gradients visuels ${ }^{15}$ joue un rôle important dans l'appréciation des variations d'échelle et des écarts de distance entre les objets représentés dans les images. Or ici seuls Tintin, Milou et le champignon sont dotés d'un facteur de différenciation pictural suffisant pour évoquer, sans ambiguité possible, la fluctuation et l'écart de taille qui les affectent, et ce en dépit du décor. Le lecteur a donc tout loisir d'interpréter la scène du champignon suivant une logique régressive.

\begin{tabular}{|l||l|l|}
\hline Lecture linéaire $=$ & $\begin{array}{l}\text { Tintin normal } \\
\text { Petit champignon }\end{array}$ & $\begin{array}{l}\text { Tintin normal } \\
\text { Énorme champignon }\end{array}$ \\
\hline \hline Lecture tabulaire* $^{*}=$ & $\begin{array}{l}\text { Énorme Tintin } \\
\text { Petit champignon }\end{array}$ & $\begin{array}{l}\text { Petit Tintin } \\
\text { Énorme champignon }\end{array}$ \\
\hline
\end{tabular}

* Cette lecture équivaut à la représentation d'un fantasme régressif.

Là où Tintin est réduit à une incapacité de représentation résultant de l'état d'angoisse provoqué par le grossissement

14 Jacques Samson, "Le champ tardien", dans Mieux vaut Tardi, sous la direction de J. Samson, Montréal, Analogon, 1989, p. 40.

15 Voir Jan B. Deregowski, "Pictorial Perception and Culture ", dans Scientific American, vol. 227, $n^{\circ}$ 5, New York, novembre 1972, p. 82 à 88 . « Density gradients are provided by any elements of uniform size: bricks in a wall or pebbles on a beach. The elements are drawn larger or smaller depending on whether they are nearer to the viewer or farther away from him " (p. 84). 
accéléré du champignon, le lecteur voit fort bien ce qu'il y a à voir. Grâce précisément aux propriétés tabulaires de la bande dessinée, la scène engendre une tache aveugle: le point de rencontre de la consécution et de la contiguïté, lieu privilégié de la lecture. À la différence de la scène de l'Observatoire, la représentation lectorielle du fantasme régressif de Tintin opère ici à travers le syntagme; il n'y a plus transfert par substitution de points de vue ('un mis à la place de l'autre), mais plutôt association par concaténation d'images, le point de vue provenant alors de l'extérieur de la scène. La contagion du sens n'est plus métaphorique mais métonymique. Hergé explore brillamment les propriétés de symbolisation de la bande dessinée; dans cette scène, il reporte vers le lecteur les difficultés d'appréciation de la réalité qui sont au cœur même de L'étoile mystérieuse.

Cette fois-ci le savoir de Tintin ne se confond plus exactement avec celui du lecteur. Ce dernier en apprend davantage à propos de la mésaventure de Tintin, en particulier qu'il fantasme une sorte de chaos adamique donnant forme et vie à ses plus intenses représentations d'angoisse. Ce champignon qui croît démesurément devant son regard pétrifié n'est-il pas le même que celui qu'il tient tout près de son ventre dans les dernières vignettes de la planche 61? Si Tintin fait l'expérience de la fin du monde dans L'étoile mystérieuse, il s'agit bel et bien, on l'aura compris, de la fin du monde de l'enfance. 